\title{
Habitat productivity and pyrethroid susceptibility status of Aedes aegypti mosquitoes in Dar es Salaam, Tanzania
}

\author{
Leah Mathias ${ }^{1}$, Vito Baraka ${ }^{2,3}$, Anitha Philbert ${ }^{1,4}$, Ester Innocent ${ }^{5}$, Filbert Francis ${ }^{2}$, Gamba Nkwengulila $^{1}$
} and Eliningaya J. Kweka, ${ }^{6,7^{*}}$

\begin{abstract}
Background: Aedes aegypti (Diptera: Culicidae) is the main vector of the dengue virus globally. Dengue vector control is mainly based on reducing the vector population through interventions, which target potential breeding sites. However, in Tanzania, little is known about this vector's habitat productivity and insecticide susceptibility status to support evidence-based implementation of control measures. The present study aimed at assessing the productivity and susceptibility status of $A$. aegypti mosquitoes to pyrethroid-based insecticides in Dar es Salaam, Tanzania.
\end{abstract}

Methods: An entomological assessment was conducted between January and July 2015 in six randomly selected wards in Dar es Salaam, Tanzania. Habitat productivity was determined by the number of female adult A. aegypti mosquitoes emerged per square metre. The susceptibility status of adult $A$. aegypti females after exposure to 0.05\% deltamethrin, $0.75 \%$ permethrin and $0.05 \%$ lambda-cyhalothrin was evaluated using the standard WHO protocols. Mortality rates were recorded after $24 \mathrm{~h}$ exposure and the knockdown effect was recorded at the time points of 10, 15, 20, 30, 40, 50 and 60 min to calculate the median knockdown times (KDT 50 and KDT 95 ).

Results: The results suggest that disposed tyres had the highest productivity, while water storage tanks had the lowest productivity among the breeding habitats Of A. aegypti mosquitoes. All sites demonstrated reduced susceptibility to deltamethrin (0.05\%) within $24 \mathrm{~h}$ post exposure, with mortalities ranging from $86.3 \pm 1.9$ (mean $\pm \mathrm{SD}$ ) to $96.8 \pm 0.9$ (mean \pm SD). The lowest and highest susceptibilities were recorded in Mikocheni and Sinza wards, respectively. Similarly, all sites demonstrated reduced susceptibility permethrin $(0.75 \%)$ ranging from $83.1 \pm 2.1 \%$ (mean $\pm S D$ ) to $96.2 \pm 0.9 \%$ (mean $\pm S D$ ), in Kipawa and Sinza, respectively. Relatively low mortality rates were observed in relation to lambda-cyhalothrin (0.05\%) at all sites, ranging from $83.1 \pm 0.7$ (mean \pm SD) to $86.3 \pm 1.4$ (mean $\pm \mathrm{SD}$ ). The median $\mathrm{KDT}_{50}$ for deltamethrin, permethrin and lambda-cyhalothrin were 24.9-30. 3 min, 24.3-34.4 min and 26.7-32.8 min, respectively. The KDT 95 were 55.2-90.9 min for deltamethrin, 54.3-94. 6 min for permethrin and 64.5-69.2 min for lambda-cyhalothrin.

Conclusions: The productive habitats for A. aegypti mosquitoes found in Dar es Salaam were water storage containers, discarded tins and tyres. There was a reduced susceptibility of $A$. aegypti to and emergence of resistance against pyrethroid-based insecticides. The documented differences in the resistance profiles of $A$. aegypti mosquitoes warrants regular monitoring the pattern concerning resistance against pyrethroid-based insecticides and define dengue vector control strategies.

Keywords: Culicidae, Aedes aegypti, Abundance, Productivity, Pyrethroid, Knockdown effect, Dar es Salaam, Tanzania

\footnotetext{
* Correspondence: kwekae@tpri.or.tz

${ }^{6}$ Department of Medical Parasitology and Entomology, Catholic University of Health and Allied Sciences, P.O. Box 1464, Mwanza, Tanzania

${ }^{7}$ Division of Livestock and Human Diseases Vector Control, Tropical Pesticides

Research Institute, Ngaramtoni, Off Nairobi Road, P.O. Box 3024, Arusha,

Tanzania

Full list of author information is available at the end of the article
} 


\section{Multilingual abstracts}

Please see Additional file 1 for translations of the abstract into the five official working languages of the United Nations.

\section{Background}

Dengue fever is a widespread vector-borne viral disease and is the tropical disease with the fastest global spread recently. Aedes spp. and dengue infections are highly prevalent in Latin America, Southern Asia and the Caribbean, and also prevalent in Sub-Saharan Africa with about 400 million dengue cases occurring annually (see Table 1) [1, 2]. The disease is the most common arbovirus infection globally, with infections and transmission occurring in at least 128 countries, putting almost four billion people at risk worldwide [2]. The number of dengue cases reported per year is 50 to 100 million cases in over 100 endemic countries [3]. Asia bears the biggest burden of dengue in the world, accounting for $70 \%$ of all cases (67 million infections), and is characterised by large belts of highly populated regions with very high conducive environments for dengue transmission [4]. By 2010, Africa had a total of 15 million cases of dengue [4]. The current global burden of the disease estimate death due to dengue to be more than 14000 people in 2010 [5].

Currently, dengue cases are being reported in Tanzania with confirmed clinical cases and dengue haemorrhagic fever in patients who attended the Bombo dispensary (Bombo, Tanga), Hai hospital (Hai, Kilimanjaro), Tanganyika plantation company (TPC) hospital (Lower Moshi, Kilimanjaro) and Kilosa district hospital (Kilosa, Morogoro) [6, 7]. Aedes aegypti mosquitoes have been found to occupy habitats in both urban and rural environments [8], in highland and lowland areas [9]. In Tanzania, Aedes mosquitoes have often been found in lowlands [8] and have also recently been found to occupy the highlands (areas defined as having an altitude of $900 \mathrm{~m}$ above sea level), but with no dengue viruses [9] as they have in the lowlands of the country $[8,10]$.

The first cases of dengue were reported between 1823 and 1870 in Zanzibar archipelago, followed by outbreaks in mainland Tanzania between 2010 and 2014 [8]. Dar es Salaam, the main commercial and administrative hub, is prone to dengue outbreaks [8]; other cases have been reported in Tanga, Morogoro and Kilimanjaro [6, 7]. The previous outbreaks in Dar es Salaam were associated with circulating dengue virus serotype 2 (DENV2) [8]. An entomological study conducted by resident researchers reported a high density of $A$. aegypti mosquitoes spreading widely throughout Dar es Salaam and in the outskirts of the city [11].

The World Health Organization (WHO) has approved the first-ever dengue vaccine recently, however, it has not been widely deployed in control programmes in many countries in Sub-Saharan Africa [12]. The current findings show that CYD-TDV is safe and efficacious for 2-14 year-olds when given as part of a three-time dose programme [13]. The vaccine efficacy for the prevention of virologically confirmed dengue cases was found to be greater than the primary endpoint threshold, which is needed for the efficacious accepted point of the vaccine. The level of efficacy for all 25 months of follow-up for study participants who had received the vaccine in Latin America had, as expected, the highest performance outcome and was hence approved by the WHO [13]. Due to the higher efficacy among vaccinated patients aged nine years and above, a licence has been obtained in several countries, which have accepted the vaccine, for patients aged 9-60 years, the group that responded the best to the vaccine $[14,15]$. There is evidence that the introduction of the CYD-TDV vaccine among adolescents in high dengue transmission zones on a routine basis will reduce the hospitalisation rate by about $10-30 \%$ [15]. Thus, A. aegypti vector control using insecticides remains the cornerstone of preventing dengue outbreaks.

Emergence and spread of vector insecticide resistance to the main classes of insecticides used in A. aegypti control is a serious operational impediment that could compromise the control of dengue and other vectorborne diseases. Although many studies have investigated the characterisation of malaria vector resistance, there is limited evidence on the $A$. aegypti insecticide resistance status in the region. Two mechanisms for insecticides resistance regarding $A$. aegypti mosquitoes are proposed: 1 ) increased activity of detoxification enzymes and glutathione S-transferases (GSTs); and (2) structural modifications in insecticide binding sites leading to a decreased affinity for the insecticide [16]. In addition, there is a suggestion of possible cross-resistance between organophosphate and pyrethroid insecticides mediated by polymorphisms in the voltage-gated sodium channel (knockdown resistance mutation) [16].

A few studies have been conducted on the effect of insecticides on natural mosquito populations in SubSaharan Africa, with one reporting reduced susceptibility to deltamethrin, lambda-cyhalothrin and propoxur in Western Africa (Dakar, Senegal) [17]. In Yaoundé, Cameroon, a study demonstrated that A. albopictus mosquitoes are probably resistant to deltamethrin, however, most of the $A$. aegypti population was shown to be susceptible to deltamethrin, propoxur and fenitrothion [18]. Little is known about the susceptibility status of the A. aegypti vector population in Eastern Africa. Elsewhere, resistance to pyrethroid-based insecticides has been shown in $A$. aegypti mosquitoes in several reports emanating from Asia, South America and Latin America 
Table 1 The Geographic distribution of spatially unique occurrence records for the Americas, Europe/Africa, and Asia/Oceania

\begin{tabular}{cll}
\hline & Country & Occurrences \\
\hline Ae.aegypti & & \\
Americas & Brazil & 5044 \\
& USA & 436 \\
& Mexico & 411 \\
& Cuba & 177 \\
& Argentina & 170 \\
& Trinidad and Tobago & 152 \\
& Venezuela & 130 \\
& Colombia & 128 \\
& Puerto Rico & 120 \\
& Peru & 89
\end{tabular}

\section{Ae. albopictus}

Americas

Brazil

USA

3441

1594

Mexico

Cayman Islands

Haiti

Guatemala

Venezuela

Colombia

Cuba

Puerto Rico

Europe/Africa

Senegal

Cameroon

Kenya

United Republic of Tanzania

Cote d'Ivoire

Nigeria

Madagascar

Gabon

Mayotte

Sierra Leone

Europe/Africa

Italy

Madagascar

Cameroon

France

Gabon

Albania

Mayotte

Greece

Israel

Lebanon
Table 1 The Geographic distribution of spatially unique occurrence records for the Americas, Europe/Africa, and Asia/Oceania (Continued)

\begin{tabular}{lll}
\hline Asia/Oceanic & Taiwan & 9490 \\
& Indonesia & 603 \\
& Thailand & 495 \\
& India & 423 \\
& Australia & 282 \\
& Viet Nam & 223 \\
& Malaysia & 112 \\
& Singapore & 44 \\
& Philippines & 36 \\
& Cambodia & 29 \\
Asia/Oceania & Taiwan & 15339 \\
& Malaysia & 186 \\
& Indonesia & 161 \\
& India & 150 \\
& Japan & 97 \\
& Thailand & 82 \\
& Singapore & 44 \\
Lao People's Democratic Republic & 26 \\
Philippines & 22 \\
Viet Nam & 18 \\
\hline
\end{tabular}

Top 10 countries in terms of occurrence records for each continent are shown for Ae.aegypti (a) and Ae.Albopictus(b) (Source: Kraemer et al, eLife 2015;4:e08347, http://dx.doi.org/10.7554/eLife.08347)

[19-22]. Thus, for conventional insecticides to remain effective for dengue vector control, it is essential that the susceptibility status of $A$. aegypti vector populations is regularly monitored.

Furthermore, dengue infection transmission relies on the productivity of female Aedes mosquitoes from breeding habitats. Habitat productivity ultimately determines the number of adult vectors. The adult female Aedes mosquitoes emerging from breeding habitats are epidemiologically important because only female adults can feed on human hosts in contrast to the mosquito larvae and pupae density [23]. Several factors can influence productivity and abundance such as female oviposition preference, habitat type and size, and vegetation cover $[23,24]$. Therefore, an estimation of the number of emerging Aedes adult mosquitoes is important to determine the productivity of mosquito breeding in their ecological habitats. Due to limited resources, the determination of the habitats that are most productive for targeted larval and adult control measures is of priority in Africa.

We adopted the WHO standard bioassay approach to investigate the resistance status of pyrethroid-based insecticides. The study aimed at investigating the 
phenotypic susceptibility status and habitat productivity of $A$. aegypti populations collected in different settings in Dar es Salaam, a city prone to seasonal dengue outbreaks.

\section{Methods}

\section{Study site}

The present study was conducted in six randomly selected wards in Dar es Salaam: Msasani, Mikocheni, Sinza, Kigogo, Kipawa and Kigamboni (Fig. 1). Dar es Salaam is one of the fastest growing cities in SubSaharan Africa, with a population growth of approximately $8 \%$ per year. It is the main administrative region and economic hub of Tanzania. The national census survey of 2012 indicates the city has a population of 4. 364541 people [25].

Dar es Salaam is located in the eastern part of the country at $6^{\circ} 52^{\prime} \mathrm{S}, 39^{\circ} 12^{\prime} \mathrm{E}$, at $55 \mathrm{~m}$ above sea level. The average temperature is $25.9{ }^{\circ} \mathrm{C}$, with the lowest and highest temperatures occurring in July - August and February - March, respectively [26]. The area is characterised by two rainy seasons: short rains (October to
December) and long rains (March to May), with a total annual average precipitation of $1148 \mathrm{~mm}$. Relative humidity is high, reaching $100 \%$ almost every night throughout the year, but falling to $60 \%$ during the day. The city is characterised by unplanned, poor sanitation and a shortage of water, which leads to storing water in vessels or containers that are potential breeding habitats for $A$. aegypti mosquitoes.

\section{Habitat productivity and abundance}

Mosquito larvae and pupae were collected from various containers and water storage vessels during the survey. The breeding habitats were recorded, including their locations and types. In relation to habitat productivity, each of the positive larval habitats, the pupae and larvae, were collected and placed in sample containers and transported to the laboratory for analysis and data recording. The pupae collected were placed in a paper cup kept in the insectary in order for the adult mosquito to emerge. The sex and species of the adults were identified based on the standard methods used by Banerjee et al. [27]. The number of females emerged was recorded for

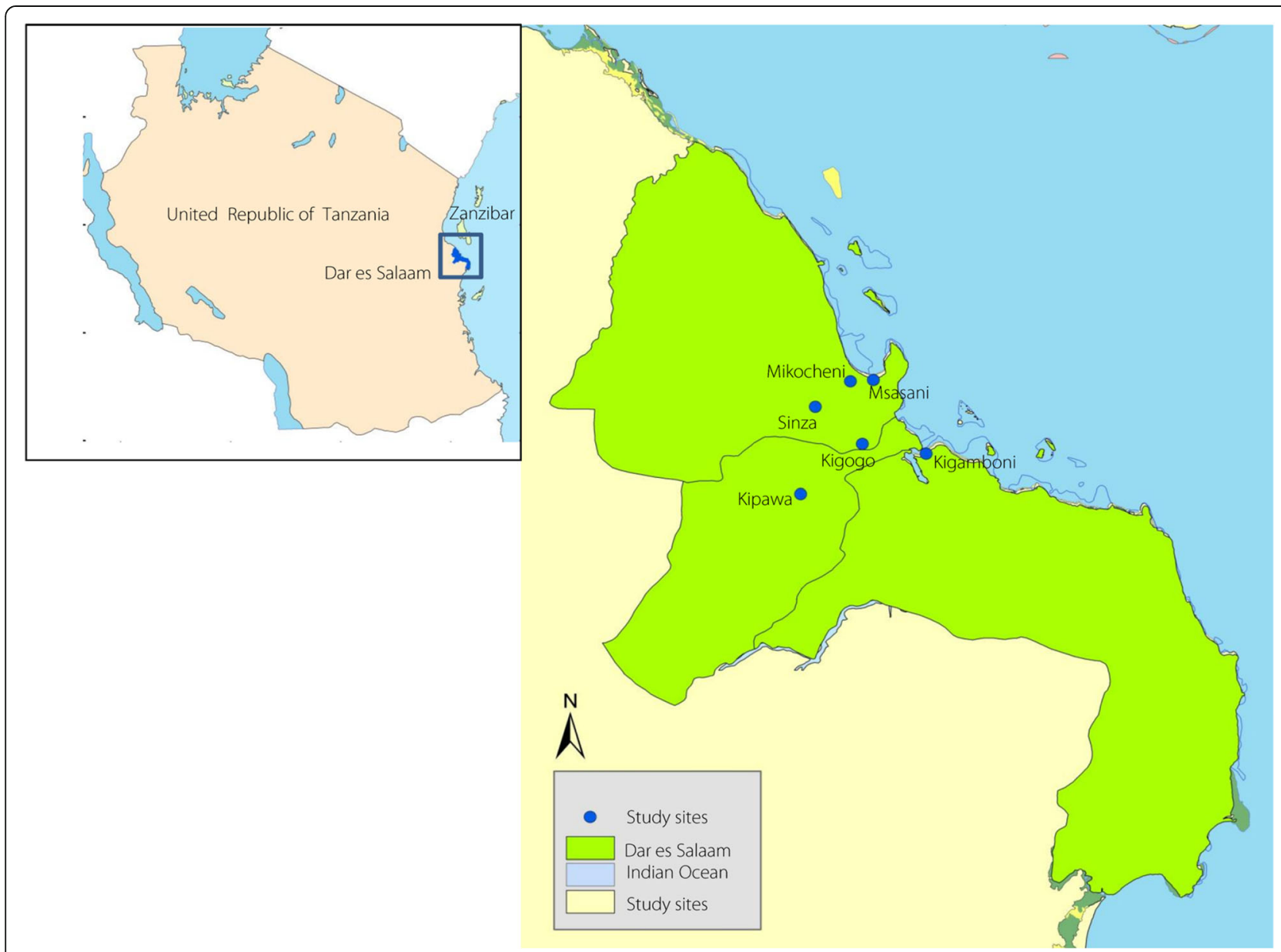

Fig. 1 A map of Dar es Salaam, Tanzania showing the sampling sites of Aedes aegypti mosquitoes 
each habitat type and site sampled. Male mosquitoes were excluded because they are not a disease vector. The surface area of each habitat type sampled was estimated in square metres. Habitat productivity was determined by calculating the number of females emerged per square metre according to previous ecological studies conducted in western Kenya highlands [28].

\section{Sample collection and mosquito rearing}

The sampling was done once a week from January to July 2015 in each selected site. The A. aegypti aquatic stages were collected using a dipper and pipette, and the geographical coordinates of each sampling site were recorded. The collected larvae and pupae were put in a container and transported immediately to the insectary where they were transferred into larvae rearing trays. The rearing was done under the standard conditions: temperature of $27{ }^{\circ} \mathrm{C} \pm 1{ }^{\circ} \mathrm{C}$ and a relative humidity of $80 \% \pm 10 \%$, with the larvae being fed cat food pellets. The pupae collected from rearing trays were kept in mosquito cages sized $30 \mathrm{~cm} \times 30 \mathrm{~cm} \times 30 \mathrm{~cm}$ until emergence. A $10 \%$ sucrose solution was prepared and used for feeding the emerged adults before the susceptibility test; this took 3-5 days depending on the number of adults needed. The emerged adult mosquitoes were sorted by separating the males from the females, and then the females were subjected to insecticide susceptibility testing.

\section{Adult bioassays for insecticide susceptibility tests}

The susceptibility test was performed according to the WHO guidelines using the emerged $A$. aegypti females, aged three days, from different sites. Batches of 20 nonblood fed female $A$. aegypti mosquitoes were aspirated in WHO holding tubes lined with untreated paper for one hour. They were then exposed to insecticides and the outcomes were recorded for each mosquito larvae sampled. Thereafter, they were provided with $10 \%$ of sucrose solution for $24 \mathrm{~h}$ after been exposed to insectcides before scoring mortality. Mosquitoes were transferred from the holding tubes to the WHO exposure tubes, which were lined with paper impregnated with the relevant pyrethroid insecticides (treatments). For each exposure, four treated tubes of the same insecticide and two control replicate tubes were used. The insecticides were deltamethrin (0.05\%), permethrin (0.75\%) and lambda-cyhalothrin $(0.05 \%)$. In the exposure kits, mosquitoes were exposed for $60 \mathrm{~min}$ and the number of mosquitoes knocked down was recorded at the following time points: 10, 15, 20, 30, 40, 50 and $60 \mathrm{~min}$. Mosquitoes were then transferred to paper cups and provided with $10 \%$ sucrose solution for recovery monitoring at $26.0{ }^{\circ} \mathrm{C} \pm 1.0{ }^{\circ} \mathrm{C}$ and $80 \% \pm 10 \%$ humidity for $24 \mathrm{~h}$. For each insecticide, there were five replicates: four treatment replicates and one control. The mortality was concluded $24 \mathrm{~h}$ post insecticide exposure.

\section{Data analysis}

Data were entered into Microsoft Excel (American Multinational Technology Company, Redmond, Washington) and transferred to SPSS version 18.0 (SPSS Inc., Chicago, IL). Larval and pupal abundances were computed using habitat type and sampling sites as factors with analysis of variance one-way analysis (ANOVA). Data were log transformed before analysis, as the data had great variations between habitats. To assess the susceptibility status, bioassays for different insecticides per site were done as according to WHO recommendations [29]: if $98-100 \%$ mosquito mortality is observed, this indicates insecticide susceptibility, mortality $<98 \%$ suggests existence of resistance that needs to be confirmed, and mortality $<90 \%$ suggests resistance [29]. Controls were also set up by systematically exposing a group of mosquitoes to untreated papers. The test results were discarded if mortality in the control group was over $20 \%$ but corrected if mortality was between 5 and 20\% using Abbot's formula [30]. To calculate the bioassays percentage knockdown times and mortality at 50 and $95 \%$ of the population $\left(\mathrm{KDT}_{50}\right.$ and $\left.\mathrm{KDT}_{95}\right)$, probit analysis incorporating regression models were applied. The habitat productivity was calculated by counting the number of adult females (disease transmitters) emerged in each habitat and divided by the surface area of that habitat by sampling site. As appropriate, means, standard errors, 95\% confidence intervals (CIs) and variances for all variables were calculated. A statistical significance level was set at $P$-value $\leq 0.05$.

\section{Results}

A total of 17461 immature mosquitoes (larvae and pupae) were obtained from the six study sites in Dar es Salaam from January to July 2015. During the collection period, discarded tins, tyres and water storage vessels were found to harbour a high abundance of $A$. aegypti larvae at the sites. The distribution of the A. aegypti larvae breeding sites are presented in Fig. 2. Larval abundance among the three habitat types (discarded tins, discarded car tyres and water storage vessels) was not statistically different (degree of freedom $(d f)=2, F$-test $(F)=1.174, P=0.311)$. Similarly, pupal abundance was not statistically different $(d f=2, F=0.919, P=0.400)$. When compared between the study sites, the larvae were equally high in all sites $(d f=5, F=1.036, P=0.397)$ with a similar trend observed for pupal abundance $(d f=5, F=1.952, P=0.086)$. 


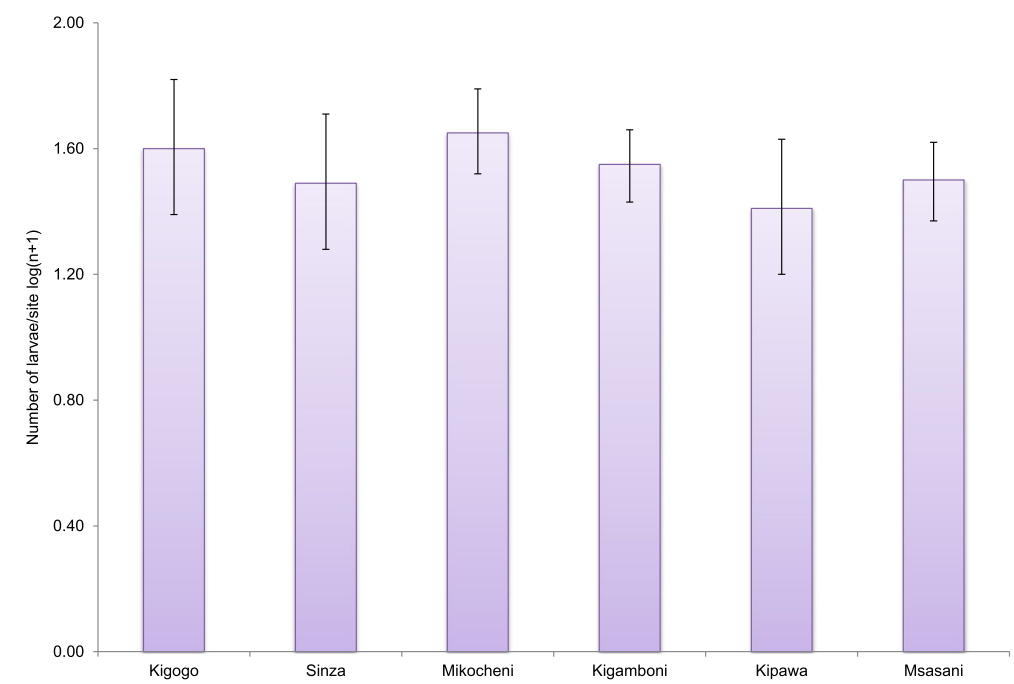

Fig. 2 Larval density as observed at the different sites in Dar es Salaam, Tanzania

\section{Aedes aegypti habitat productivity}

Figures 3 and 4 illustrate the productivity of $A$. aegypti mosquitoes at the different study sites. Adult productivity was highest in disposed tyres $(P<0.002)$, while water storage tanks had the lowest productivity $(P<0.0004)$. Our findings suggest a consistent pattern of productivity across the sites (see Fig. 3).

\section{Susceptibility status of adult $A$. aegypti mosquitoes to insecticides \\ Adult bioassays}

The susceptibility status of populations of $A$. aegypti mosquitoes to different concentrations of pyrethroid insecticides is shown in Table 2.
Resistance to $0.05 \%$ deltamethrin was detected in the Msasani and Mikocheni collected strains and the mean mortality rates $24 \mathrm{~h}$ post exposure were 87.5 and $86.3 \%$, respectively. The samples collected from the remaining sites were found to have suspected resistance to $0.05 \%$ deltamethrin that needs further investigation.

The results of $0.75 \%$ permethrin bioassays suggest resistance in almost all sites with the exception of two sites (Sinza and Kigamboni), where resistance is suspected with a mortality rate of above $90 \%$ but less than $98 \%$. The mean mortality rates $24 \mathrm{~h}$ post exposure for $0.75 \%$ permethrin ranged between 83.1 and $96.2 \%$ (see Table 2). The mortality rates pertaining to Aedes mosquitoes collected from Kigamboni (91.2\%) and Sinza (96.2) were relatively high and classified as suspected resistance.

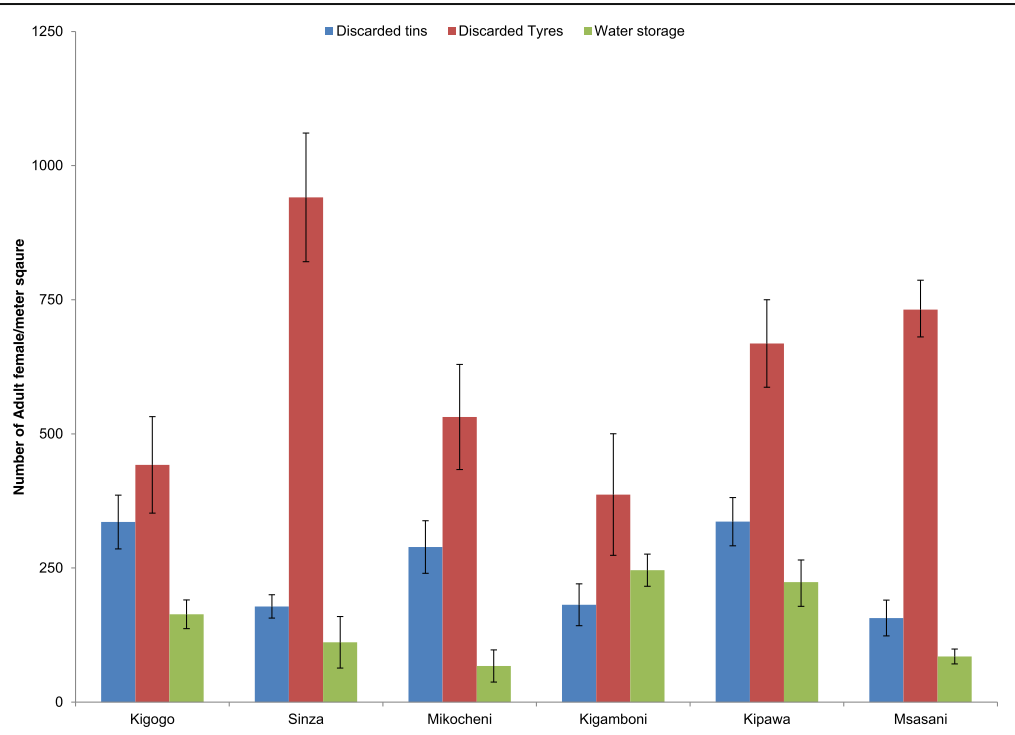

Fig. 3 Habitat productivity of Aedes aegypti mosquitoes at the three breeding habitats in the six wards in Dar es Salaam, Tanzania 


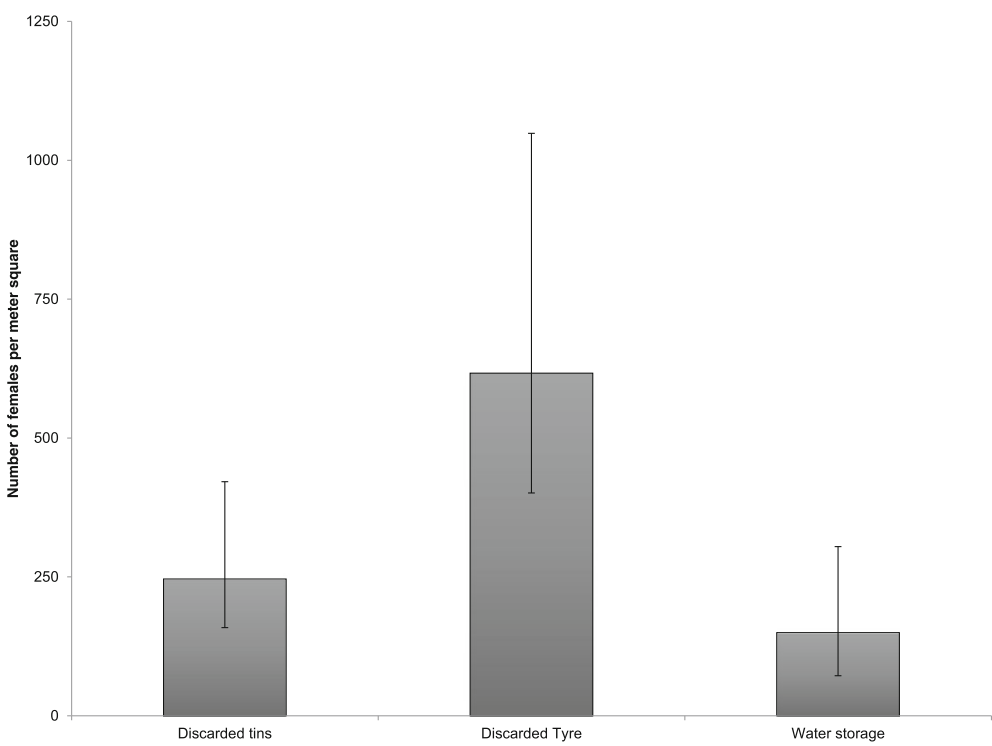

Fig. 4 Female Aedes aegypti habitat productivity at the different breeding habitats in Dar es Salaam, Tanzania

For A. aegypti mosquitoes subjected to $0.05 \%$ lambda-cyhalothrin, the mortality recorded ranged from 83.1 to $86.3 \%$. Full susceptibility to $0.05 \%$ lambda-cyhalothrin was recorded in mosquitoes from all six sites (see Table 2).

\section{Knockdown times}

For $0.05 \%$ deltamethrin bioassays, the $\mathrm{KDT}_{50}$ ranged from 24.9 to $30.3 \mathrm{~min}$, while $\mathrm{KDT}_{95}$ ranged from 55.2 to $90.9 \mathrm{~min}$. The highest $\mathrm{KDT}_{50}(30.3 \mathrm{~min})$ was observed in mosquitoes collected in Kigamboni (see Table 2).

Table 2 Knock-down times and mortality rates of field collected Aedes aegypti mosquitoes exposed to 0.05\% lambdacyhalothrin 0.75\% permethrin and 0.05\% lambdacyhalothrin using WHO standard bioassay

\begin{tabular}{|c|c|c|c|c|c|c|c|c|c|}
\hline Insecticide & Site & $(N)$ & Replicates & Mean mortality (\%) $\pm S D$ & KDT50 (Min.) & $95 \% \mathrm{Cl}$ & KDT95 (Min.) & $95 \% \mathrm{Cl}$ & Status \\
\hline \multirow[t]{6}{*}{ 0.05\% Deltamethrin } & Kigogo & 160 & 8 & $90.6 \pm 1.8$ & 30 & $24.6-36.8$ & 90.9 & $64.6-178.9$ & $\mathrm{R}^{*}$ \\
\hline & Kipawa & 160 & 8 & $92.5 \pm 0.9$ & 24.9 & $21.7-28.3$ & 55.2 & $45.8-73.7$ & $R^{*}$ \\
\hline & Msasani & 160 & 8 & $87.5 \pm 1.3$ & 29.9 & $24.0-30.1$ & 78.7 & $64.1-105.9$ & R \\
\hline & Sinza & 160 & 8 & $96.8 \pm 0.9$ & 26 & $22.8-29.4$ & 60.1 & $49.8-80.1$ & $R^{*}$ \\
\hline & Mikocheni & 160 & 8 & $86.3 \pm 1.9$ & 24.9 & $21.3-28.6$ & 58.5 & $47.4-82.3$ & R \\
\hline & Kigamboni & 160 & 8 & $91.9 \pm 1.1$ & 30.3 & $27.0-33.9$ & 65.9 & $55.1-86.4$ & $\mathrm{R}^{*}$ \\
\hline \multirow[t]{6}{*}{ 0.75\% Permethrin } & Kigogo & 160 & 8 & $86.8 \pm 1.2$ & 30.9 & $28.1-34.0$ & 68.1 & $58.2-85.4$ & R \\
\hline & Kipawa & 160 & 8 & $83.1 \pm 2.1$ & 34.4 & $30.6-39.0$ & 94.6 & $74.9-35.9$ & R \\
\hline & Msasani & 160 & 8 & $85.0 \pm 1.3$ & 32.1 & $27.9-36.9$ & 71.2 & $57.4-102.5$ & $\mathrm{R}$ \\
\hline & Sinza & 160 & 8 & $96.2 \pm 0.9$ & 24.3 & $21.0-27.6$ & 54.3 & $44.8-73.4$ & $R^{*}$ \\
\hline & Mikocheni & 160 & 8 & $88.1 \pm 1.4$ & 33.7 & $28.1-41.1$ & 89.3 & $65.3-164.2$ & $\mathrm{R}$ \\
\hline & Kigamboni & 160 & 8 & $91.2 \pm 1.0$ & 29.3 & $27.0-31.7$ & 67.1 & $58.6-80.4$ & $R^{*}$ \\
\hline \multirow[t]{6}{*}{ 0.05\% Lambdacyhalothrin } & Kigogo & 160 & 8 & $86.3 \pm 1.4$ & 29.2 & $27.9-30.6$ & 67.2 & $61.8-74.1$ & $\mathrm{R}$ \\
\hline & Kipawa & 160 & 8 & $85.6 \pm 2.1$ & 26.7 & $22.7-31.0$ & 64.5 & $51.5-94.1$ & $\mathrm{R}$ \\
\hline & Msasani & 160 & 8 & $85.0 \pm 0.7$ & 32.8 & $31.5-34.3$ & 69 & $63.9-75.7$ & $\mathrm{R}$ \\
\hline & Sinza & 160 & 8 & $83.8 \pm 0.7$ & 30.7 & $27.5-34.3$ & 76 & $62.8-101.1$ & R \\
\hline & Mikocheni & 160 & 8 & $84.4 \pm 0.6$ & 27.9 & $25.6-30.4$ & 69.2 & $59.8-84.2$ & R \\
\hline & Kigamboni & 160 & 8 & $83.1 \pm 0.7$ & 29.3 & 27.9-30.7 & 69.6 & $64.8-77.2$ & $\mathrm{R}$ \\
\hline
\end{tabular}

$N$ number of samples, $C l$ confidence interval, $S D$ standard deviation, KDT knock-down time, KDT50 time taken for $50 \%$ of the test mosquitoes to knock down, KDT95 time taken for $95 \%$ of the test mosquitoes to knock down. S; Full susceptible (observed mortality $98-100 \%$ ) R*; Suspected resistance needs to be confirmed (mortality 90-97\%) and R; Resistance (observed mortality < 90\%) 
The $\mathrm{KDT}_{50}$ values were comparable across the sites, ranging from 24.3 to $34.4 \mathrm{~min}$, while the $\mathrm{KDT}_{95}$ ranged from 54.3 to $94.6 \mathrm{~min}$ for $0.75 \%$ permethrin. The longest $\mathrm{KDT}_{50}(34.4 \mathrm{~min})$ was recorded in Kipawa [95\% CI: 30.6-39.0] (see Table 2).

For $0.05 \%$ lambda-cyhalothrin, the observed $\mathrm{KDT}_{50}$ and $\mathrm{KDT}_{95}$ ranged between 26.7 to $32.8 \mathrm{~min}$ and 64.5 to $69.2 \mathrm{~min}$, respectively. Overall, it took a long time for mosquitoes to be knocked down (32.8 min) by lambdacyhalothrin in Msasani compared with the other sites (see Table 2).

\section{Discussion}

Despite the development of a recent dengue vaccine $\left(\right.$ Dengvaxia $\left.^{\circ}\right)$ and its endorsement by the WHO, the vaccine is still unavailable in Sub-Saharan countries. Therefore, vector control remains the cornerstone of dengue prevention and control. Other insecticides are an integral part of vector control, however, the paucity of data on the susceptibility status of $A$. aegypti mosquitoes could compromise the effectiveness of dengue vector control campaigns. In this study, we examine data on A. aegypti productivity and the mosquito population's pyrethroid susceptibility status in Dar es Salaam, a city experiencing frequent outbreaks of dengue fever.

The breeding habitats included in this study were those which were positively infested with $A$. aegypti larvae. The habitats found to have Aedes larvae were disposed tyres, water storage containers and discarded tins. Tyres and water storage containers located outdoors appeared to be the most stable breeding habitats for $A$. aegypti mosquitoes. Discarded tyres are used for various purposes including fencing and in car garages, and they are found in many places. In addition, plastic water storage vessels of various sizes also contribute to the abundance of breeding habitats. Most households keep water storage vessels due to water scarcity and irregular water shortages. Our findings are consistent with a previous study that also reported that discarded tyres have a high abundance of $A$. aegypti larvae in Dar es Salaam [11]. Similarly, a recent study found that most of the water storage vessels in most of the households in the same setting harboured larvae or pupae of Aedes spp. [8].

Regarding $A$. aegypti female adult productivity, our data suggest a similar pattern of habitat productivity across all sites. However, tyres had the highest productivity, while water storage containers had the lowest productivity among the observed breeding habitats. This also compares with other findings that documented high productivity in discarded car tyres $[8,11]$. However, contrary to our finding, a study conducted in the Philippines and Malaysia on habitat productivity demonstrated that domestic containers were the most productive and targeted for $A$. aegypti control, which have added value to dengue control in these countries $[6,7]$. Other breeding habitats have also been documented, including selfwatering pots and domestic waste disposal containers, which was not observed in the present study [11]. Nonetheless, our data suggest similar female A. aegypti productivity in habitats as also reported in other settings $[24,27,31]$. This demonstrates that for effective reduction and elimination of the most productive $A$. aegypti mosquitoes, campaigns should also target water storage containers to reduce mosquito density. Our study was, however, limited by the fact that seasonal productivity and influence of other climatic variables could not be explored, and this is worth exploring in future studies.

The susceptibility test results of dengue vectors generally demonstrated that $A$. aegypti populations from Dar es Salaam subjected to lambda-cyhalothrin had the highest level of resistance in all six study sites, with a mortality rate of less than $86 \%$, which shows increased resistance. However, the WHO recommends further investigation on the mechanisms and distribution of resistance to be undertaken if the observed mortality is between 90 and 96\% [29]. Of the three insecticides tested for susceptibility, deltamethrin showed the highest mortality rate, while permethrin showed a moderate mortality rate and lambdacyhalothrin showed the lowest mortality rate. Similar results regarding resistance to pyrethroids were found by Marcombe et al. [20, 21]. The level of susceptibility varied according to the insecticide used and sites. A. aegypti resistance to lambda-cyhalothrin seems to have increased across the sites compared with the other insecticides examined in this study. The reason as to why lambda-cyhalothrin had developed higher levels of Insecticide resistance compared to the others is merely associated with the intensive use of lambdacyhalothrin in conventionally treated bed nets in Tanzania [32]. However, possible cross-resistance with insecticides used in malaria control is also speculated. To our knowledge, this is the first study to demonstrate the susceptibility status of $A$. aegypti against different registered pyrethroids in Tanzania and there are limited data to compare our findings with. In other countries, studies have found detailed mechanisms that are involved in the different insecticides resistance, which is also needed to be done in Tanzania for strategic control of $A$. aegypti as in Thailand and Brazil [33, 34].

Mosquito populations from Mikocheni, Kipawa, Kigogo and Kigamboni showed high resistance to all three insecticides. Mikocheni had the lowest resistance, but mosquitoes from Sinza showed a susceptibility to deltamethrin and permethrin, with mortalities of 97 and $96 \%$ 
respectively, and indicators of resistance to lambdacyhalothrin. The high resistance among Aedes mosquito populations can be attributed to the prolonged use of these insecticides in controlling mosquitoes domestically, such as with sprays, coils and long-lasting insecticidal nets (LLINs). Pyrethroids have also been widely used in agriculture [35-38]. The frequent use of insecticides for vector control has led to increasing concerns over the development of insecticide resistance of these vectors on the environment and human health, which can compromise vector control strategies. The rapid spread of the Aedes vector due to transportability of either adult Aedes through vehicles or dried but viable eggs through containers could influence the spread and outbreak of dengue infections in non-endemic areas of Tanzania.

\section{Conclusions}

This study has for first time shown an evidence-based spread of pyrethroid-based insecticide resistance in $A$. aegypti populations in Dar es salaam, Tanzania. This calls for a countrywide survey to be conducted to assess the susceptibility status of $A$. aegypti for better management of vector and all arboviruses transmitted by this vector.

The findings suggest that the high habitat productivity recorded in discarded tyres needs to be targeted for outbreak prevention and for controlling dengue fever infections. Our data suggest that $A$. aegypti populations in most of the sites in Dar es Salaam are fully resistant to permethrin and lambda-cyhalothrin, while deltamethrin demonstrated suspected resistance. The documented differences in the resistance profiles of $A$. aegypti mosquitoes warrant regular monitoring to elucidate the pattern concerning resistance against pyrethroid-based insecticides and define dengue vector control strategies.

\section{Additional files}

Additional file 1: Multilingual abstracts in the five official working languages of the United Nations. (PDF 894 kb)

Additional file 2: The habitat larval and pupae abundance, physical chemical properties of habitat in different wards of Dar-es-salaam , Tanzania. (XLS 94 kb)

\section{Abbreviations}

Cl: Confidence Interval; COSTECH: Commission for Science and Technology; $d f$ : Degree of Freedom; F: F-test; $\mathrm{KDT}_{50}$ : Knockdown time for $50 \%$ of the population; KDT 95 : Knockdown time for $95 \%$ of the population; NRF: National Research Foundation; WHO: World Health Organisation

\section{Acknowledgements}

The authors acknowledge the contributions of Dr Yahya Athuman, Mr Bernard Malongo, Dr Patrick Tungu and Mr Wema Sudi (NIMR Amani) for their valuable efforts to support with the materials needed for the implementation of the project. Staff members of the University of Dar es Salaam and Institute of
Traditional Medicine are thanked for their assistance during the execution of the laboratory work.

\section{Funding}

This study had some financial support from LM's and VB's families. Some support was also provided by the Institute of Traditional Medicine at Muhimbili University College of Health, and other materials were funded through the Tanzania Commission for Science and Technology (COSTECH) and National Research Foundation (NRF) (grant no. TZ-RSA/JRP/RG.2013.08).

\section{Availability of data and materials}

The dataset supporting the conclusions of this article is included with the article as an additional file (Additional file 2).

\section{Authors' contributions}

LM, VB, GN, EJK and AP conceived the idea for the study. LM and El designed and coordinated the bioassays and field sample collection. LM, EJK and FF performed the statistical analysis. LM, VB and EK wrote the paper. All authors read and approved the final version.

\section{Competing interests}

The authors declare that they have no competing interests.

\section{Consent for publication}

Not applicable.

Ethics approval and consent to participate

Not applicable.

\section{Author details}

${ }^{1}$ Department of Zoology and Wildlife Conservation, College of Natural and Applied Sciences, University of Dar es Salaam, P.O. Box 35165, Dar es Salaam, Tanzania. ${ }^{2}$ National Institute for Medical Research, Tanga Medical Research Centre, P.O. Box 5004Tanga Urban, Tanga, Tanzania. ${ }^{3}$ Global Health Institute, University of Antwerp, Gouverneur Kinsbergencentrum, Doornstraat 331B-2610, Wilrijk, Belgium. ${ }^{4}$ Mkwawa University College of Education, Private Bag, Iringa, Tanzania. ${ }^{5}$ Institute of Traditional Medicine, Muhimbili University of Health and Allied Sciences, P.O. Box 65001, Dar es Salaam, Tanzania.

${ }^{6}$ Department of Medical Parasitology and Entomology, Catholic University of Health and Allied Sciences, P.O. Box 1464, Mwanza, Tanzania. ${ }^{7}$ Division of Livestock and Human Diseases Vector Control, Tropical Pesticides Research Institute, Ngaramtoni, Off Nairobi Road, P.O. Box 3024, Arusha, Tanzania.

Received: 27 May 2016 Accepted: 12 May 2017

Published online: 09 June 2017

\section{References}

1. Rogers DJ, Wilson AJ, Hay SI, Graham AJ. The global distribution of yellow fever and dengue. In Advances in Parasitology. Vol 62. Simon I. Hay AG, David JR, editors. London: Academic Press; 2006. pp. 181-220.

2. Bhatt $S$, Gething PW, Brady OJ, Messina JP, Farlow AW, Moyes $C L$, et al. The global distribution and burden of dengue. Nature. 2013;496:504-7.

3. Dengue (http://www.who.int/denguecontrol/en/), Accessed 10 Jan 2016.

4. Murray NE, Quam MB, Wilder-Smith A. Epidemiology of dengue: past, present and future prospects. Clin Epidemiol. 2013;5:299-309.

5. Vos T, Flaxman AD, Naghavi M, Lozano R, Michaud C, Ezzati M, et al. Years lived with disability (YLDs) for 1160 sequelae of 289 diseases and injuries 1990 - 2010: a systematic analysis for the Global Burden of Disease Study 2010. Lancet. 2013;380:2163-96.

6. Saifur RGM, Hassan AA, Dieng H, Salmah MRC, Saad AR, Satho T. Temporal and spatial distribution of dengue vector mosquitoes and their habitat patterns in Penang Island, Malaysia. J Am Mosq Control Assoc. 2013:29:33-43.

7. Edillo FE, Roble ND, Otero II ND. The key breeding sites by pupal survey for dengue mosquito vectors, Aedes aegypti (Linnaeus) and Aedes albopictus (Skuse), in Guba, Cebu City, Philippines. Southeast Asian J Trop Med Public Health. 2012:43:1365

8. Mboera LE, Mweya CN, Rumisha SF, Tungu PK, Stanley G, Makange MR, et al. The risk of denque virus transmission in Dar es Salaam, Tanzania during an epidemic period of 2014. PLoS Negl Trop Dis. 2016;10:e0004313. 
9. Hertz JT, Lyaruu LJ, Ooi EE, Mosha FW, Crump JA. Distribution of Aedes mosquitoes in the Kilimanjaro Region of northern Tanzania. Pathog Glob Health. 2016;110:108-12.

10. Chipwaza B, Mugasa JP, Selemani M, Amuri M, Mosha F, Ngatunga SD, et al. Dengue and Chikungunya fever among viral diseases in outpatient febrile children in Kilosa district hospital, Tanzania. PLoS Negl Trop Dis. 2014;8:e3335.

11. Philbert A, ljumba JN. Preferred breeding habitats of Aedes aegypti (DipteraCulicidae) mosquito and its public health implications in Dares Salaam, Tanzani. J Environ Res Mang. 2013;4:344-51.

12. Pitisuttithum $P$, Bouckenooghe $A$. The first licensed dengue vaccine: an important tool for integrated preventive strategies against dengue virus infection. Expert Rev Vaccines. 2016;15:795-8.

13. Capeding MR, Tran NH, Hadinegoro SRS, Ismail HIHJM, Chotpitayasunondh $T$, Chua MN, et al. Clinical efficacy and safety of a novel tetravalent dengue vaccine in healthy children in Asia: a phase 3, randomised, observermasked, placebo-controlled trial. Lancet;384:1358-65.

14. Wilder-Smith A, Massad E. Age specific differences in efficacy and safety for the CYD-tetravalent dengue vaccine. Expert Rev Vaccines. 2016;15:437-41.

15. Wilder-Smith A, Vannice KS, Hombach J, Farrar J, Nolan T. Population perspectives and World Health Organization recommendations for CYD-TDV dengue vaccine. J Infect Dis. 2016;214:1796-1799.

16. Martins AJ, Lima JBP, Peixoto AA, Valle D. Frequency of Val1016lle mutation in the voltage-gated sodium channel gene of Aedes aegypti Brazilian populations. Trop Med Int Health. 2009;14:1351-5.

17. Dia I, Diagne CT, Ba Y, Diallo D, Konate L, Diallo M. Insecticide susceptibility of Aedes aegypti populations from Senegal and Cape Verde Archipelago. Parasit Vectors. 2012;5:238.

18. Kamgang B, Marcombe S, Chandre F, Nchoutpouen E, Nwane P, Etang J, et al. Insecticide susceptibility of Aedes aegypti and Aedes albopictus in Central Africa. Parasit Vectors. 2011:4:1-8.

19. Saavedra-Rodriguez K, Urdaneta-Marquez L, Rajatileka S, Moulton M, Flores A, Fernandez-Salas I, et al. A mutation in the voltage-gated sodium channel gene associated with pyrethroid resistance in Latin American Aedes aegypti. Insect Mol Biol. 2007:16:785-98.

20. Marcombe S, Carron A, Darriet F, Etienne M, Agnew P, Tolosa M, et al Reduced efficacy of pyrethroid space sprays for dengue control in an area of Martinique with pyrethroid resistance. Am J Trop Med Hyg. 2009:80:745-51.

21. Marcombe S, Mathieu RB, Pocquet N, Riaz M-A, Poupardin R, Sélior S, et al. Insecticide resistance in the dengue vector Aedes aegypti from Martinique: distribution, mechanisms and relations with environmental factors. PLoS One. 2012;7:e30989.

22. Jirakanjanakit N, Rongnoparut $P$, Saengtharatip S, Chareonviriyaphap T, Duchon S, Bellec C, et al. Insecticide susceptible/resistance status in Aedes (Stegomyia) aegypti and Aedes (Stegomyia) albopictus (Diptera: Culicidae) in Thailand during 2003-2005. J Econ Entomol. 2007;100:545-50.

23. de Brito Arduino M. Assessment of Aedes aegypti pupal productivity during the Dengue Vector Control Program in a Costal Urban Centre of São Paulo State, Brazil. J Insects. 2014:2014:9. doi:10.1155/2014/301083.

24. Mohan S, Banerjee S, Mohanty SP, Saha GK, Aditya G. Assessment of pupal productivity of Aedes and co-occurring mosquitoes in Kolkata, India. Southeast Asian J Trop Med Public Health. 2014;45:1279-91.

25. NBS. United Republic of Tanzania: 2012 Population and Housing Census. Dar es salaam: Central Census Office Tanzania; 2012.

26. Trpis M. Seasonal changes in the larvel populations of Aedes aegypti in two biotopes in Dar es Salaam, Tanzania. Bull World Health Organ. 1972;47:245.

27. Banerjee S, Aditya G, Saha GK. Pupal productivity of dengue vectors in Kolkata, India: implications for vector management. Indian J Med Res. 2013;137:549.

28. Kweka EJ, Zhou G, Lee M-C, Gilbreath TM, Mosha F, Munga S, et al. Evaluation of two methods of estimating larval habitat productivity in western Kenya highlands. Parasit Vectors. 2011;4:1-9.

29. WHOPES. Test procedures for insecticide resistance monitoring in malaria vector mosquitoes. Geneva: World Health Organisation; 2013.

30. Abbott W. A method of computing the effectiveness of an insecticide. J Am Mosq Control Assoc. 1987;3:302-3.

31. Banerjee S, Mohan S, Saha N, Mohanty SP, Saha GK, Aditya G. Pupal productivity \& nutrient reserves of Aedes mosquitoes breeding in sewage drains \& other habitats of Kolkata, India: implications for habitat expansion \& vector management. Indian J Med Res. 2015;142:S87.
32. Mashauri FM, Kinung'hi SM, Kaatano GM, Magesa SM, Kishamawe C, Mwanga JR, et al. Impact of indoor residual spraying of lambda-Cyhalothrin on malaria prevalence and anemia in an Epidemic-Prone District of Muleba, North-Western Tanzania. Am J Trop Med Hyg. 2013:88:841-9.

33. Plernsub S, Saingamsook J, Yanola J, Lumjuan N, Tippawangkosol P, Sukontason $\mathrm{K}$, et al. Additive effect of knockdown resistance mutations, S989P, V1016G and F1534C, in a heterozygous genotype conferring pyrethroid resistance in Aedes aegypti in Thailand. Parasit Vectors. 2016:9:1-7.

34. Bona ACD, Chitolina RF, Fermino ML, de Castro PL, Weiss A, Lima JBP, et al. Larval application of sodium channel homologous dsRNA restores pyrethroid insecticide susceptibility in a resistant adult mosquito population. Parasit Vectors. 2016;9:1-14.

35. Philbert A, Lyantagaye SL, Nkwengulila G. A review of agricultural pesticides use and the selection for resistance to insecticides in malaria vectors. Adv Entomol. 2014;2014

36. Silva APB, Santos JMM, Martins AJ. Mutations in the voltage-gated sodium channel gene of anophelines and their association with resistance to pyrethroids - a review. Parasit Vectors. 2014;7:1-14.

37. Nkya TE, Poupardin R, Laporte F, Akhouayri I, Mosha F, Magesa S, et al. Impact of agriculture on the selection of insecticide resistance in the malaria vector Anopheles gambiae: a multigenerational study in controlled conditions. Parasit Vectors. 2014;7:1-12.

38. Yadouléton A, Badirou K, Agbanrin R, Jöst H, Attolou R, Srinivasan R, et al. Insecticide resistance status in Culex quinquefasciatus in Benin. Parasit Vectors. 2015:8:1-6.

\section{Submit your next manuscript to BioMed Central and we will help you at every step:}

- We accept pre-submission inquiries

- Our selector tool helps you to find the most relevant journal

- We provide round the clock customer support

- Convenient online submission

- Thorough peer review

- Inclusion in PubMed and all major indexing services

- Maximum visibility for your research

Submit your manuscript at www.biomedcentral.com/submit
) Biomed Central 